\title{
Ring opening and cycloadditions of novel fused 1,2,4-triazines
}

\author{
Zsuzsanna Riedl $^{\mathrm{a} *}$, György Hajós ${ }^{\mathrm{a}}$, Péter Kövér ${ }^{\mathrm{a}}$, and Gert Kollenz \\ ${ }^{a}$ Institute of Chemistry, Chemical Research Center, Hungarian Academy of Sciences, $\mathrm{H}-1525$ \\ Budapest, POBox 17, Hungary , ${ }^{b}$ Institute of Organic Chemistry, University of Graz, \\ Heinrichstr. 28, A-8020 Graz, Austria \\ E-mail : zriedl@chemres.hu
}

\section{Dedicated to Professor Gábor Bernáth on his $70^{\text {th }}$ birthday}

(received 14 Jan 03; accepted 06 Mar 03; published on the web 14 Mar 03)

\begin{abstract}
Ring opening of two ring transformation products of furo[2,3-e]pyrido[1,2-b][1,2,4]triazinium salt, i.e. a pyrrolo[2,3-e]pyrido[1,2-b][1,2,4]triazine derivative and a zwitterionic pyrido[1,2-b]pyridazino[3,4-e][1,2,4]triazine compound has been investigated. The pyrrole-fused tricycle when reacted with some dienophiles resulted, unlike its zwitterionic pyridazine analogue, only in Michael addition and/or subsequent ring opening. Reaction of the methylated $N$ methylpyrrolo[2,3-e]pyrido[1,2-b][1,2,4]triazinium derivative and dimethyl pyrido[1,2$b]$ pyridazino[3,4-e][1,2,4]triazinium salt with secondary amines afforded new hetaryldienes which, in some cases, were successfully subjected to Diels-Alder reaction to give regular cycloadducts.
\end{abstract}

Keywords: Fused 1,2,4-triazines, zwitterion, hetaryldiene, cycloaddition, ring opening

\section{Introduction}

In the course of our activity in the area of ring transformation reactions of some polyfused triazines we have described that 2-arylfuro[2,3-e]pyrido[1,2-b][1,2,4]triazinium salts (1) readily react with nucleophiles and undergo ring transformations to a pyrrole-fused tricyclic ring system (2) or differently substituted pyridazines, e.g. to the zwitterionic pyrido[1,2-b]pyridazino[3,4-e]$[1,2,4]$ triazine $(3)^{1}$. We have also reported that these tricyclic derivatives $(2,3)$ can be selectively alkylated to quaternary salts (4, and 5 , respectively) $)^{2}$. Furthermore, reactivity of the zwitterionic 3 has also been studied in detail: we found that this compound when reacted with acetylenedicarboxylic ester can undergo dipolar cyclization. Reaction with tetracyanoethylene resulted in oxydative dimerization to yield a stable radical cation, whereas reaction with tosyl isocyanate yielded Michael addition products ${ }^{3}$. All these results prompted us to explore the 
reactivity of the pyrrole-fused analogue 2 with particular respect to possible cycloaddition and ring opening transformations.

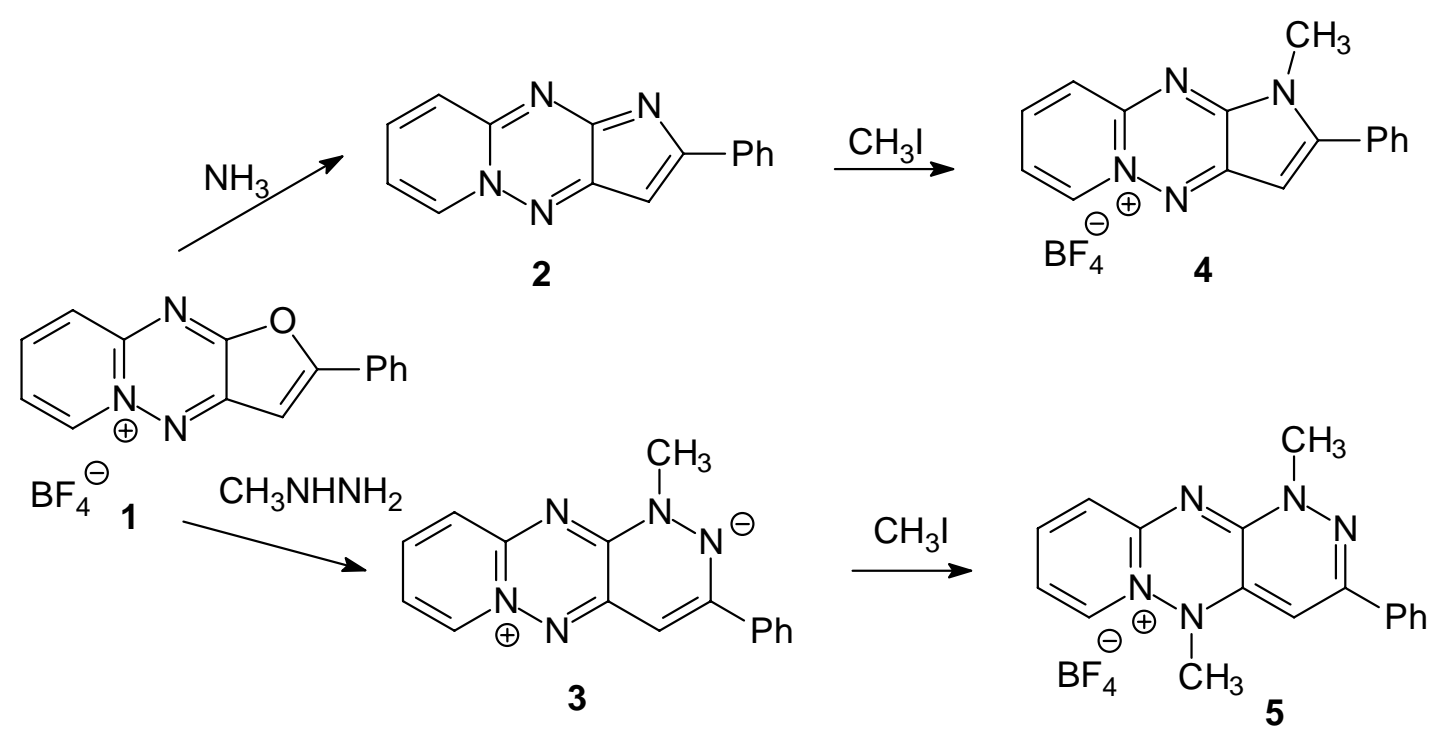

\section{Results and Discussion}

The pyrrole-fused tricyclic compound was reacted - for comparison with our earlier results with the analogous pyridazine derivative $\mathbf{3}$ - with dimethyl acetylenedicarboxylate, tosyl isocyanate and tetracyanoethylene. Interestingly enough, all these reagents resulted in Michael-additions and no cycloaddition or transformation of any other type was found. Thus, both with tetracyanoethylene and tosyl isocyanate the regular Michael adducts (6 and $\mathbf{7}$, respectively) were isolated in acceptable yields. A more interesting transformation was observed during the reaction of $\mathbf{2}$ and dimethyl acetylenedicarboxylate: although Michael addition also took place in this case, because of the presence of traces of water during the work up, the addition intermediate underwent hydrolytic ring opening to $\mathbf{8}$ which was obtained in good yield.
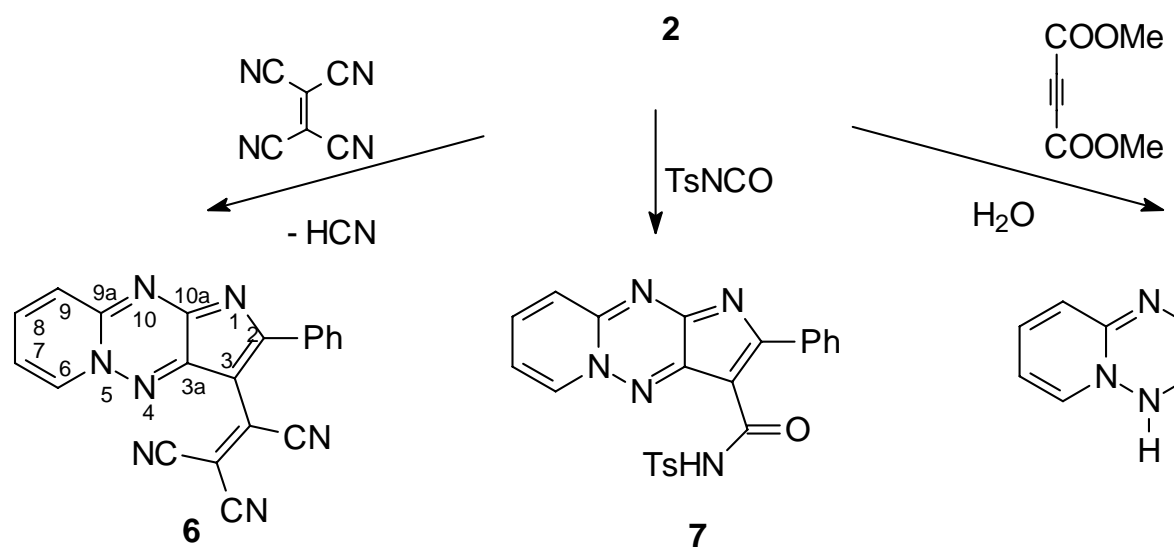<smiles>O=C(N[AsH3])c1c(-c2ccccc2)nc2nc3ccccn3nc1-2</smiles>

7<smiles>COC(=O)/C=C(\C=C(/N=c1nc2ccccn2[nH]c1=O)c1ccccc1)C(C)=O</smiles>

8 


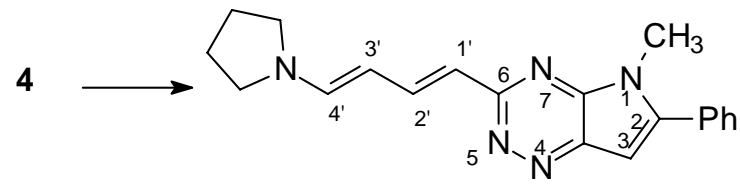

9

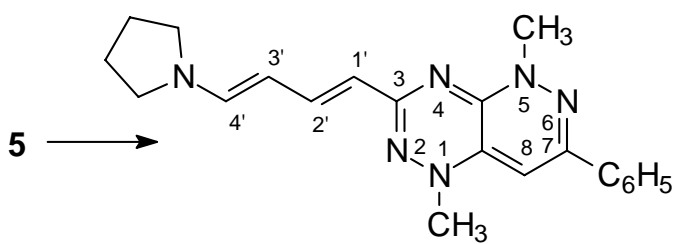

10

Both positively charged methylated salts (1-methyl-2-phenylfuro[2,3-e]pyrido[1,2b][1,2,4]triazinium tetrafluoroborate $\mathbf{4}$ and 1,5-trimethylpyrido[1,2-b]pyridazino[3,4$e][1,2,4]$ triazinium tetrafluoroborate 5) reacted with secondary amines. In these transformations -like in several cases found by us earlier ${ }^{4,5}$ - the pyridine moiety underwent ring opening to yield new hetaryldienes, $\mathbf{9}$ and 10, respectively. The new dienes, in accordance with the general experience $e^{6-8}$, had a trans-trans geometry as supported unambiguously by the coupling constants of the diene protons in the ${ }^{1} \mathrm{H}-\mathrm{NMR}$ spectrum.

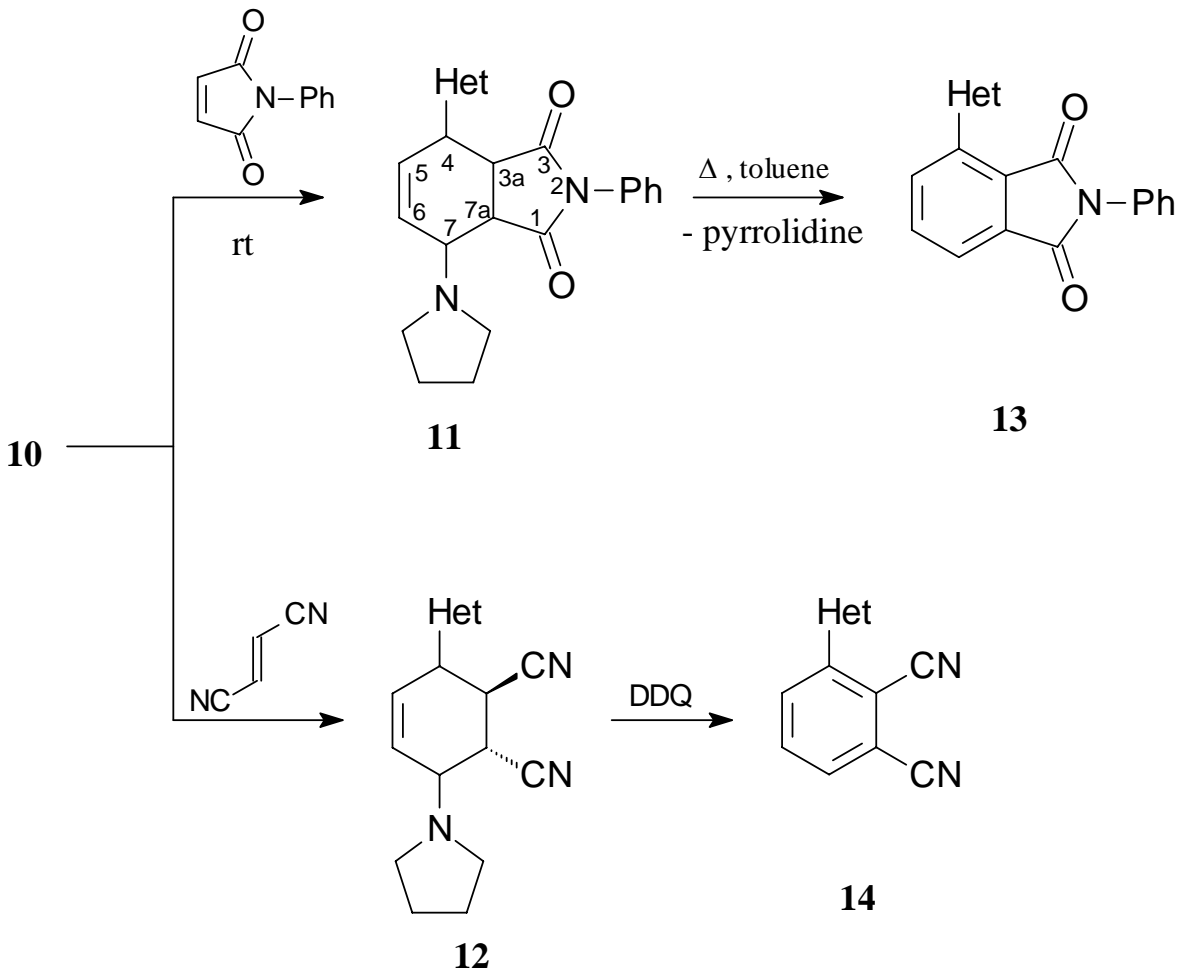

While our efforts to carry out cycloaddition reactions with $\mathbf{9}$ failed, diene $\mathbf{1 0}$ reacted relatively easily with $\mathrm{N}$-phenylmaleinimide and fumaronitrile to yield regular cycloadducts containing a partially saturated benzene ring (11 and 12, respectively). Aromatization of the benzene moiety was carried out with both cycloadducts. In the case of cycloadduct $\mathbf{1 1}$ simple heating in toluene was enough to facilitate elimination of pyrrolidine and a subsequent spontaneous oxidation to give the corresponding isoindol-2,7-dione derivative $\mathbf{1 3}$. The similar 
transformation with the fumaronitrile adduct was carried out by oxidation with DDQ to yield the hetarylphtalonitrile 14.

\section{Conclusions}

Transformations observed in these studies provided access to several new fused heterocyclic derivatives. These compounds seem to be promising candidates for biological (particularly DNA intercalation $^{9}$ - determined by measurement of the $T_{\mathrm{m}}$ point $^{10}$ - and multidrug resistance inhibitory ${ }^{11}$ ) tests which are now in progress in the frame of COST B16 action.

\section{Experimental Section}

General Procedures. Melting points were determined by a Büchi apparatus and are uncorrected. The IR spectra were recorded with a Nicolet Magna 750 FT-IR, spectrophotometers; the NMR spectra were recorded with a Varian UNITY INOVA spectrometer (400 $\mathrm{MHz}$ for ${ }^{1} \mathrm{H}$ and 100 $\mathrm{MHz}$ for ${ }^{13} \mathrm{C}$ ) and Varian VXR200 spectrometer (200 MHz for $\left.{ }^{1} \mathrm{H}\right)$.

\section{2-Cyano-3-(2-phenylpyrrolo[2,3-e]pyrido[1,2-b][1,2,4]triazin-3-yl)but-2-enedinitrile (6).} Tetracyanoethylene $(0.13 \mathrm{~g}, 1 \mathrm{mmol})$ was added to a suspension of 2-phenylpyrrolo[2,3$e]$ pyrido[1,2-b][1,2,4]triazine $(2,0.25 \mathrm{~g}, 1 \mathrm{mmol})$ in dichloromethane $(5 \mathrm{~mL})$, and the mixture was stirred at rt for $1 \mathrm{~h}$. A solid separated which was filtered off and recrystallized from acetonitrile to give $0.15 \mathrm{~g}$ (43\%) of red crystals, mp 250-252 ${ }^{\circ} \mathrm{C}$. IR (KBr): 2216, 1581, 1519, 1502, 1481, 1455, 1409, 1242, 1184, 1153, 1138, 1127, 1102, $782 \mathrm{~cm}^{-1} ;{ }^{1} \mathrm{H}$ NMR $\delta\left(\mathrm{CDCl}_{3}\right)$ : 7.68 (m, 3H, H-phenyl), 7.71 (t, 1H, $J=7,9$ Hz, H-7), 8.01 (m, 2H, H- phenyl), 8.18 (t, 1H, $J=$ 9, 9.3, Hz H-8), 8.31 (d, 1H, $J=9.3$, Hz H-9), 9.11 (d, 1H, $J=7 \mathrm{~Hz}, \mathrm{H}-6$ ); ${ }^{13} \mathrm{C}$ NMR $\delta$ (DMSO$\left.\mathrm{d}_{6}\right)$ : 82.9(C=C), 99.3(C=C), 112.9(CN), 113.2(CN), 113.6(CN), 120.8(C-7), 126.7(C-9), 128.5(C-phenyl), 130.5(C-3), 130.6, 132.2 and 133.1(C-phenyl), 137.3(C-8), 138.1(C-6), 144.6(C-9a), 145.3 (C-2), 154.9(C-3a), 179.4(C-10a). Anal. Calcd. for $\mathrm{C}_{20} \mathrm{H}_{9} \mathrm{~N}_{7}$ (347.35): C, 69.15; H, 2.61; N, 28.23. Found: C, 68.63; H, 2.68; N, 28.10.

\section{4-Methyl- $N$-(2-phenylpyrrolo[2,3-e]pyrido[1,2-b][1,2,4] triazin-3-carbonyl)-benzenesulfonamide}

(7). The reagent ( $p$-toluenesulfonyl isocyanate, $1.0 \mathrm{~mL}, 6.6 \mathrm{mmol}$ ) was added to the compound of 2-phenylpyrrolo[2,3-e]pyrido[1,2-b][1,2,4]triazine (2, $0.25 \mathrm{~g}, 1 \mathrm{mmol})$ and the mixture was stirred at rt for $1 \mathrm{~h}$. The reaction mixture was diluted with diethylether (10 $\mathrm{mL})$ and a yellow solid separated which was filtered off and recrystallized from DMF to give $0.32 \mathrm{~g}$ (72\%) of product, mp 275-278 ${ }^{\circ} \mathrm{C}$. IR (KBr): 3261, 3069, 1678, 1581, 1485, 1461, 1429, 1346, 1333, 1161, 1140, 1131, 1078, 1020, 750, $666 \mathrm{~cm}^{-1}$; ${ }^{1} \mathrm{H}$ NMR $\delta$ (DMSO-d $)$ : 7.40 (d, 1H, H-9), 7.5 (m, 3H, H-phenyl), 7.55 (m, 1H, H-7), 7.76 (m, 1H, H-8), 7.92 (m, 4H, H-tolyl), 8.2 (m, 2H, Hphenyl), 9.26 (d, $1 \mathrm{H}, J=7 \mathrm{~Hz}, \mathrm{H}-6), 11.5(\mathrm{NH}) ;{ }^{13} \mathrm{C}$ NMR $\delta$ (DMSO-d (D) $^{21.8, ~ 119.6, ~ 126.8, ~}$ 
128.0, 128.2, 129.6, 130.5, 131.4, 134.5, 135.8, 137.2, 137.9, 144.1, 144,6, 146.5, 160.9, 162.6. Anal. Calcd. for $\mathrm{C}_{23} \mathrm{H}_{17} \mathrm{~N}_{5} \mathrm{O}_{3} \mathrm{~S}$ (443.50): C, 62.29; H, 3.86; N, 15.79. Found: C, 62.20; H, 3.95; N, 15.80 .

Dimethyl 2-[2-(3-oxo-3,4-dihydropyrido[1,2-b][1,2,4]triazin-2-ylideneamino)-2-phenylvinyl]-but-2-enedioate (8). Dimethyl acetylenedicarboxylate $(0.3 \mathrm{~mL}, 2.4 \mathrm{mmol})$ was added to a suspension of 2-phenylpyrrolo[2,3-e]pyrido[1,2-b][1,2,4]triazine (2, $0.5 \mathrm{~g}, 2 \mathrm{mmol})$ in dichloromethane $(10 \mathrm{~mL})$, and the mixture was stirred at rt for $6 \mathrm{~h}$. The reaction mixture was evaporated and the product was isolated by column chromatography (Alumuniumoxide, chloroform) and recrystallized from ethanol to give yellow crystals (0.15 g, 18\%), mp 210$215^{\circ} \mathrm{C}$. IR (KBr): 3087, 3045, 2949, 1732, 1675, 1609, 1552, 1529, 1490, 1436, 1298, 1226 , 1157, 1136, $770 \mathrm{~cm}^{-1}$; ${ }^{1} \mathrm{H}$ NMR $\delta\left(\mathrm{CDCl}_{3}\right): 3.19$ (s, 3H, H-COMe), 3.82 (s, 3H, H-COMe), 5.60 (s, 1H, H-3'), 6.25 (s, 1H, H-5'), 6.95 (t, 1H, J = 7.2, 9 Hz, H-7), 7.40 (d, 1H, J = 9.3, Hz H-9), 7.60 (t, 1H, $J=9$, 9.3, Hz H-8), 7.60-7.20 (m, 5H, H-phenyl), 8.32 (d, 1H, $J=7.2$ Hz, H-6) 12.3 $(\mathrm{NH}) ;{ }^{13} \mathrm{C} \mathrm{NMR} \delta\left(\mathrm{CDCl}_{3}\right): 51.7,52.3,98.0,98.5,113.5,123.7,128.1,130.0,136.4,136.6$, 137.2, 145.6, 151.0, 151.5, 152.5, 159.9, 164.1, 168.2. Anal. Calcd. for $\mathrm{C}_{21} \mathrm{H}_{18} \mathrm{~N}_{4} \mathrm{O}_{5}$ (406.41): C, 62.06; H, 4.46; N, 13.79. Found: C, 61.84; H, 4.62; N, 13.43.

\section{1-Methyl-2-phenyl-6-(4-pyrrolidin-1-yl-buta-1,3-dienyl)-1H-pyrrolo[2,3-e][1,2,4]triazine}

(9). Pyrrolidine $(0.7 \mathrm{~mL}, 8.4 \mathrm{mmol})$ was added to a solution of 1-methyl-3-phenylpyrrolo[2,3e]pyrido[1,2-b][1,2,4]triazinium tetrafluoroborate (4, $0.7 \mathrm{~g}, 2 \mathrm{mmol})$ in acetonitrile $(10 \mathrm{~mL})$, and the mixture was stirred at $\mathrm{rt}$ for $1 \mathrm{~h}$. A solid separated which was filtered off and recrystallized from acetonitrile to give reddish crystals (0.34 g, 51\%), mp 148-150 ${ }^{\circ} \mathrm{C}$. IR (KBr): 2970,1624 , 1604, 1581, 1418, 1397, 1369, 1264, 1094, 980, $748 \mathrm{~cm}^{-1}$; ${ }^{1} \mathrm{H}$ NMR $\delta\left(\mathrm{CDCl}_{3}\right): 1.93$ (m, 4H, Hpyrrolidine), 3.28 (m, 4H, H- pyrrolidine), 3.76 (s, 3H, H-1-Me), 5.28 (dd, 1H, J=12.8, $11.4 \mathrm{~Hz}$, H-2'), 6.53 (d, 1H, J = 14.5 Hz, H-4'), 6.81 (s, 1H, H-3), 6.90 (d, 1H, J = 12.8 Hz, H-1'), 7.78 (dd, $1 \mathrm{H}, J=14.5,11.4 \mathrm{~Hz}, \mathrm{H}-3$ ) $)$ 7.50-7.55 (m, 5H, H-phenyl); ${ }^{13} \mathrm{C}$ NMR $\delta\left(\mathrm{CDCl}_{3}\right)$ : 25.2, 29.0, 48.9, 98.7, 99.9, 115.8, 125.2, 128.9, 129.3, 130.7, 139.4, 143.6, 144.0, 146.6, 160.4. Anal. Calcd. for $\mathrm{C}_{20} \mathrm{H}_{21} \mathrm{~N}_{5}$ (331.41): C, 72.48; H, 6.39; N, 21.13. Found: C, 72.16; H, 6.21; N, 21.18. 1,5-Dimethyl-7-phenyl-3-(pyrrolidin-1-yl-buta-1,3-dienyl)-1,5-dihydropyridazino[3,4-e][1,2,4]triazine (10). Pyrrolidine $(2 \mathrm{~mL}, 24 \mathrm{mmol})$ was added to a solution of 1,5-dimethyl-3phenylpyrido[1,2-b]pyridazino[3,4-e][1,2,4]triazinium tetrafluoroborate 5 (4 g, $10.6 \mathrm{mmol})$ in acetonitrile $(20 \mathrm{~mL})$, and the mixture was stirred at rt. A violet solid separated which was filtered off and recrystallized from acetonitrile to give $2.56 \mathrm{~g}(67 \%)$ of product, mp 188-90 ${ }^{\circ} \mathrm{C} .{ }^{1} \mathrm{H}$ NMR $\delta\left(\mathrm{CDCl}_{3}\right.$ ): 1.88 (m, 4H, H-pyrrolidine), 2.82 (s, 3H, H-1-Me), 3.19 (m, 4H, H- pyrrolidine), 3.31 (s, 3H, H-5-Me), 4.75 (s, 1H, H-8), 5.04 (dd, 1H, $J=12.6,11.5$ Hz, H-2'), 5.41 (d, 1H, J = 14.5 Hz, H-4'), 6.70 (d, 1H, $J=12.6$ Hz, H-1'), 7.03 (dd, 1H, $J=14.5,11.5$ Hz, H-3'), 7.33-7.60 (m, $5 \mathrm{H}, \mathrm{H}$-phenyl); ${ }^{13} \mathrm{C}$ NMR $\delta\left(\mathrm{CDCl}_{3}\right)$ : 25.2, 39.1, 39.3, 48.8, 84.6, 98.3, 114.2, 125.2, 128.3, 129.1, 136.2, 136.8, 141.1, 142.4, 153.0, 158.3.

4-(1,5-Dimethyl-7-phenyl-1,5-dihydropyridazino[3,4-e][1,2,4]triazin-3-yl)-7-pyrrolidin-1-yl3a,4,7,7a-tetrahydroisoindol-1,3-dione (11). A mixture of $N$-phenylmaleinimide (110 mg, 0.64 mmol), 1,5-dimethyl-7-phenyl-3-(pyrrolidin-1-yl-buta-1,3-dienyl)-1,5-dihydropyridazino[3,4- 
e][1,2,4]triazine $(\mathbf{1 0}, 200 \mathrm{mg}, 0.55 \mathrm{mmol})$, and toluene $(10 \mathrm{~mL})$ was stirred at $\mathrm{rt}$. Orange-red crystals separated which were filtered off and washed with ether to give $226 \mathrm{mg}$ (76\%) of product, mp 166-8 ${ }^{\circ} \mathrm{C} .{ }^{1} \mathrm{H}$ NMR $\delta\left(\mathrm{CDCl}_{3}\right): 1.85$ (m, 4H, H-pyrrolidine), 2.72 (m, 4H, Hpyrrolidine), 2.79 (s, 3H, H-5'-Me), 2.90-2.96 (m, 2H, H-4, H-7), 3.20 (s, 3H, H-1'-Me), 3.54 (dd, $1 \mathrm{H}, J=9.5,7.0 \mathrm{~Hz}, \mathrm{H}-3 \mathrm{a}$ or H-7a), 3.86 (dd, 1H, $J=9.0,6.0 \mathrm{~Hz}, \mathrm{H}-3 \mathrm{a}$ or H-7a), 4.63 (s, 1H, H-8'), 6.10 (dt, $1 \mathrm{H} J=9.5,3.5$ Hz, H-6), 6.36 (dt, $1 \mathrm{H}, J=9.5,3.0 \mathrm{~Hz}, \mathrm{H}-5$ ), 7.23-7.26, 7.29-7.42 and 7.50-7.54 (m, 10H, H-phenyl); ${ }^{13} \mathrm{C}$ NMR $\delta\left(\mathrm{CDCl}_{3}\right):$ 24.5, 40.3, 40.4, 41.7, 43.9, 44.3, 54.7, 64.8, 86.2, 126.4, 127.7, 129.3, 129.5, 129.8, 130.4, 131.8, 133.1, 137.0, 143.3, 155.5, 158.4, 158.6, 175.5, 176.6. Anal. Calcd. for $\mathrm{C}_{31} \mathrm{H}_{31} \mathrm{~N}_{7} \mathrm{O}_{2}$ (533.62): C, 69.77; H, 5.86; N, 18.37. Found: C, 69.64; H, 5.86; N, 18.28.

3-(1,5-Dimethyl-7-phenyl-1,5-dihydropyridazino[3,4-e][1,2,4]triazin-3-yl)-6-pyrrolidin-1-ylcyclohexene-1,2-dicarbonitrile (12). A mixture of 1,5-dimethyl-7-phenyl-3-(pyrrolidin-1-ylbuta-1,3-dienyl)-1,5-dihydropyridazino[3,4-e][1,2,4]triazine $\quad(\mathbf{1 0}, 400 \mathrm{mg}, \quad 1.1 \mathrm{mmol})$, fumaronitrile (100 mg, $1.28 \mathrm{mmol})$ and dichloromethane $(10 \mathrm{~mL})$ was stirred at $\mathrm{rt}$ for $4 \mathrm{~d}$. The mixture was evaporated and the residue subjected to column chromatography on silica (with chloroform as an eluent). The main fraction gave $220 \mathrm{mg}$ (45\%) of pale yellow product, mp 220-222 ${ }^{\circ} \mathrm{C} .{ }^{1} \mathrm{H}$ NMR $\delta\left(\mathrm{CDCl}_{3}\right): 2.69$ (m, 4H, H-pyrrolidine), 2.71 (s, 3H, H-1'-Me), 2.73 (m, 4H, H-pyrrolidine), 3.11 (dd, 1H, $J=11.0,6.0 \mathrm{~Hz}, \mathrm{H}-1$ ), 3.13 (s, 3H, H-5'-Me), 2.97 (ddd, 1H, $J$ = 6.0, 4.5, $1.5 \mathrm{~Hz}, \mathrm{H}-6$ ), 3.63 (ddd, $1 \mathrm{H}, J=10.2,2.0,1.5 \mathrm{~Hz}, \mathrm{H}-3$ ), 3.98 (dd, $1 \mathrm{H}, J=11.0,10.2$ Hz, H-2), 4.70 (s, 1H, H-8'), 5.77 (ddd, $1 \mathrm{H}, J=10.3,4.5,2.0 \mathrm{~Hz}, \mathrm{H}-5$ ), 5.84 (dt, $1 \mathrm{H}, J=10.3$, $1.5 \mathrm{~Hz}, \mathrm{H}-4) ;{ }^{13} \mathrm{C} \mathrm{NMR} \delta\left(\mathrm{CDCl}_{3}\right): 24.4,30.6,32.1,39.1,40.1,41.4,48.4,58.2,86.3,117.6$, 119.7, 125.5, 127.6, 128.0, 128.7, 129.8, 135.7, 142.7, 155.4, 157.8, 158.5. Anal. Calcd. for $\mathrm{C}_{25} \mathrm{H}_{26} \mathrm{~N}_{8}$ (438.53): C, 68.47; H, 5.98; N, 25.55. Found: C, 68.23; H, 5.92; N, 25.54.

4-(1,5-Dimethyl-7-phenyl-1,5-dihydropyridazino[3,4-e[1,2,4]triazin-3-yl)-2-phenylisoindol-1,3dione (13). A solution of 4-(1,5-Dimethyl-7-phenyl-1,5-dihydropyridazino[3,4-e][1,2,4]triazin-3yl)-7-pyrrolidin-1-yl-3a,4,7,7a-tetrahydroisoindol-1,3-dione (11, $300 \mathrm{mg}, 0.56 \mathrm{mmol})$ in toluene $(5 \mathrm{~mL})$ was refluxed for $2 \mathrm{~h}$. Evaporation of the mixture gave a residue which was crystallized from acetonitrile to give $182 \mathrm{mg}$ (70\%) of dark gray crystals, mp 268-70 ${ }^{\circ} \mathrm{C}$. ${ }^{1} \mathrm{H}$ NMR $\delta\left(\mathrm{CDCl}_{3}\right): 2.83$ (s, 3H, H-1'-Me), 3.20 (s, 3H, H-5'-Me), 4.78 (s, 1H, H-8'), 7.35-7.58 (m, 10H, H-phenyl), 7.73 (t, 1H, $J=7.5 \mathrm{~Hz}, \mathrm{H}-6), 7.88$ (dd, 1H, $J=7.5,1.0 \mathrm{~Hz}, \mathrm{H}-5), 7.92$ (dd, $1 \mathrm{H}, J=$ 7.5, $1.0 \mathrm{~Hz}, \mathrm{H}-7) ;{ }^{13} \mathrm{C} \mathrm{NMR} \delta\left(\mathrm{CDCl}_{3}\right): 39.1,39.6,86.0,123.9,125.3,126.7,128.0,128.5$, 129.0, 129.6, 131.8, 132.8, 134.0, 134.2, 135.6, 135.7, 142.6, 154.9, 155.9, 157.8, 165.5, 166.7.

Anal. Calcd. for $\mathrm{C}_{27} \mathrm{H}_{20} \mathrm{~N}_{6} \mathrm{O}_{2}$ (460.49): C, 70.42; H, 4.38; N, 18.25. Found: C, 70.31; H, 4.28; N, 18.31 .

3-(1,5-Dimethyl-7-phenyl-1,5-dihydropyridazino[3,4-e][1,2,4]triazin-3-yl)phtalonitrile (14). A mixture of 3-(1,5-dimethyl-7-phenyl-1,5-dihydropyridazino[3,4-e][1,2,4]triazin-3-yl)-6pyrrolidin-1-yl-cyclohexene-1,2-dicarbonitrile (12, $260 \mathrm{mg}, 0.6 \mathrm{mmol}$ ), DDQ (297 mg, 1.3 $\mathrm{mmol})$ and toluene $(10 \mathrm{~mL})$ was refluxed for $1 \mathrm{~h}$. The mixture was evaporated, the residue treated with dichloromethane and the insoluble material was removed. The filtrate was evaporated and the residue was subjected to chromatography on silica (with a chloroform- 
methanol 50:1 mixture as an eluent). The main fraction gave $110 \mathrm{mg}$ (49\%) of green crystalline product, mp 239-41 ${ }^{\circ} \mathrm{C} .{ }^{1} \mathrm{H}$ NMR $\delta\left(\mathrm{CDCl}_{3}\right): 2.90$ (s, 3H, H-5'-Me), 3.55 (s, 3H, H-1'-Me), 4.80 (s, 1H, H-8'), 7.39 (m, 3H, H-phenyl), 7.56 (m, 2H, H-phenyl), 7.60 (t, 1H, J = $8.0 \mathrm{~Hz}, \mathrm{H}-5$ ), 7.72 (dd, $1 \mathrm{H}, J=8.0,1.5 \mathrm{~Hz}, \mathrm{H}-6$ ), 8.14 (dd, $1 \mathrm{H}, J=8.0,1.5 \mathrm{~Hz}, \mathrm{H}-4$ ). Anal. Calcd. for $\mathrm{C}_{21} \mathrm{H}_{15} \mathrm{~N}_{7}$ (365.39): C, 69.03; H, 4.14; N, 26.83. Found: C, 68.90; H, 3.91; N, 26.78.

\section{Acknowledgments}

Thanks are due to financial support provided by Österreichisches Ost- und Südosteuropa-Institut. Research fund OTKA T 33105 as well as 1/047 NKFP MediChem is gratefully acknowledged.

\section{References}

1. Juhász-Riedl, Zs.; Hajós, Gy.; Gács-Baitz, E.; Kollenz, G.; Messmer, A. Chem. Ber. 1990, 123, 1415.

2. Juhász-Riedl, Zs.; Hajós, Gy.; Gács-Baitz, E.; Kollenz, G.; Messmer, A. Chem. Ber. 1991, 124, 1477.

3. Riedl, Zs.; Hajós, Gy.; Messmer, A.; Rockenbauer, A.; Korecz, L.; Kollenz, G.; Fabian, W. M. F.; Peters, K.; Peters, E. M. J. Chem. Soc., Chem. Commun. 1997, 757.

4. Kotschy, A.; Hajós, Gy.; Messmer, A. J. Org. Chem. 1995, 60, 4919.

5. Hajós, Gy.; Kotschy, A. Acta Chim. Slov. 1998, 45, 285.

6. Messmer, A.; Hajós, Gy.; Timári, G. Tetrahedron 1992, 48, 8451.

7. Sanders, G. M.; van Dijk, M.; van der Plas, H. C. J. Heterocycl. Chem. 1982, 19, 797.

8. Sanders, G. M.; van Dijk, M.; van der Plas, H. C. J. Heterocycl. Chem. 1983, 20, 407.

9. Hajós, Gy.; Riedl, Zs.; Molnár, J.; Szabó D. Drugs of the Future 2000, 25, 1, 47.

10. Héléne, C. Proc. Nat. Acad. Sci. USA 1994, 81, 3297.

11. Sharples, D.; Molnár, J.; Szabó, D.; Hajós, Gy.; Riedl, Zs.; Csányi, D. Arch. Pharm. 2001, 331, 269. 